\title{
Filtragem de rejeitos de minério de ferro visando à sua disposição em pilhas
}

\author{
Filtering of iron ore tailings \\ for its disposal in stockpiles
}

\section{Nilton Caixeta Guimarães \\ Engenheiro, MSC - VALE \\ nilton.caixeta@vale.com \\ George E.S. Valadão \\ Professor, Doutor, \\ Departamento de Engenharia de Minas \\ Universidade Federal de Minas Gerais \\ gvaladao@demin.ufmg.br}

\section{Antônio E.C. Peres}

Professor, Ph.D.

Departamento de Engenharia Metalúrgica e de Materiais - Universidade Federal de Minas Gerais aecperes@demet.ufmg.br

\section{Resumo}

A metodologia tradicional de disposição de rejeitos em forma de polpa requer grandes áreas para disposição e apresenta grande impacto ambiental e, por conseguinte, as empresas têm dificuldade na aquisição de novos licenciamentos. A proximidade de áreas urbanas e a pressão pública têm despertado a necessidade de se buscarem alternativas a essa metodologia. Uma solução viável para essa questão contribuirá com o processo de licenciamento ambiental, garantindo a sobrevivência da mineração no Quadrilátero Ferrífero. Foram realizados ensaios de filtragem em bancada com amostras de rejeitos nas principais tecnologias de filtragem: a vácuo, sob pressão, capilar e hiperbárica. Os resultados mostraram que, com exceção das lamas, é possível filtrar os rejeitos com uma boa taxa de filtragem e umidade adequada para empilhamento. A filtragem das lamas sob pressão resultou em taxa unitária de filtragem relativamente baixa e alta umidade da torta (próximo a $20 \%$ ). A torta derivada das lamas poderia ser misturada à torta de rejeitos grossos e/ou o estéril da mina para permitir o seu empilhamento, porém requerendo estudos geotécnicos complementares.

Palavras-chave: Filtragem, rejeitos, empilhamento.

\begin{abstract}
The traditional method for the disposal of slurry in tailing dams requires large areas, with large environmental impact and therefore makes it difficult to acquire new licenses. The proximity of urban areas, together with public pressure, has raised the need to seek alternatives for this methodology. A viable solution to this issue will contribute to the environmental licensing process for new projects, ensuring the survival of mining in the Iron Quadrangle. Laboratory-scale filtration tests were conducted with tailing's samples using the main filtering technologies: vacuum filtration, pressure, hyperbaric and capillary filters. The results showed that with the exception of the slimes, it is possible to filter the tailings from the processing plants with a good filtration rate and residual moisture suitable for stacking. The filtration of slimes under pressure resulted in low filtration rate and high residual cake moisture (around $20 \%$ ). The resulting cake could be mixed with the coarse tailings and / or mine waste to facilitate its stacking, although it requires further geotechnical studies.
\end{abstract}

Keywords: Filtering, tailings, stockpiles. 


\section{Introdução}

O aumento do volume de rejeitos gerados nas atividades mineradoras, impulsionado pela explotação crescente de jazidas com mais baixo teor metálico, tem acentuado a necessidade de barragens cada vez maiores para a contenção dos rejeitos.

É o caso, por exemplo, do Projeto de Itabiritos de Vargem Grande (ITMI VGR), em Minas Gerais, com start up previsto para 2013. O minério (ROM), com $44 \%$ de ferro e $36 \%$ de sílica, será originado inicialmente da mina de Abóboras, para produção de 10 milhões de toneladas por ano de pellet feed. Com recuperação mássica de 46\%, demandará uma grande área para a disposição dos rejeitos (geração de $1.026 \mathrm{t} / \mathrm{h}$ de rejeito de flotação e 546t/h de lamas). Novos projetos para beneficiamento de itabiritos pobres, no Quadrilátero Ferrífero, também estão em desenvolvimento para serem implantados até 2016. É o caso dos Projetos ITM S (Mina do Pico), Itabiritos

\section{Material e métodos}

As amostras, para os estudos, são provenientes das principais usinas de minério de ferro do Quadrilátero Ferrífero: Alegria, Brucutu, Cauê, Conceição, Fábrica Nova / Timbopeba, Pico e Vargem Grande. Verificou-se que a maior geração de rejeitos, no total, das usinas amostradas é proveniente das etapas de flotação e deslamagem (aproximadamente 34\% cada), seguidas pela etapa de concentra-

\section{Resultados e discussão}

A Figura 1 apresenta as distribuições granulométricas dos principais rejeitos das usinas amostradas.

Para as lamas, a curva granulométrica da usina de Brucutu apresenta-se muito diferente das demais usinas, sendo mais fina na faixa $<10 \mu \mathrm{m}$ e mais grossa nas frações $>30 \mu \mathrm{m}$. O espessador de lamas de Brucutu recebe, também, além
Pobres de Conceição, Projeto Apollo e Itabiritos Mariana.

As preocupações ambientais envolvidas nesse processo e a pressão da opinião pública têm dificultado o licenciamento de áreas para a construção de novas barragens, despertando a necessidade de se investigarem novos métodos, para a sua disposição. Segundo Baginsk (2009), além da dificuldade de disponibilização de novas áreas, para a disposição de rejeitos, devido à proximidade, cada vez maior de áreas residenciais, existe uma dificuldade adicional, em Minas Gerais, para aprovação de licenciamentos de barragens maiores de 800 hectares.

A disposição dos rejeitos em pilhas pode ser uma alternativa viável. A polpa previamente adensada seria filtrada e a torta produzida seria disposta em pilhas (em separado, em mistura ou em co-disposição com o estéril da mina). Leduk e Smith (2003) apresentam, em artigo, as diversas técnicas empregadas de co-dis-

ção magnética (valor próximo a 21\%).

A distribuição granulométrica das amostras foi determinada utilizando-se peneiramento e granulômetro a laser.

Os ensaios de filtragem foram realizados com concentração de sólidos na alimentação de $60 \%$, em massa (exceto para as lamas, onde a concentração de sólidos foi de $40 \%$ ), e temperatura ambiente de polpa. O dimensionamento de posição de rejeitos. A filtragem dos rejeitos proporciona, também, o aumento de recuperação de água de processo da usina, reduzindo o consumo de água nova. Além da dificuldade de licenciamento de barragens, existe, também, uma dificuldade adicional nos novos projetos de aquisição de outorga para direito de uso de recurso hídrico.

A filtragem de rejeitos é uma prática usual nas minerações do deserto de Atacama, na região norte do Chile, para reduzir as perdas de água, devido a sua escassez na região. $\mathrm{Na}$ mina de ouro de $\mathrm{La}$ Coipa, que fica próxima da cidade de Copiapó, todo o rejeito do beneficiamento é filtrado e transportado por correia transportadora até a área de empilhamento.

Esse trabalho visa a comparar as diversas tecnologias de filtragem, disponíveis no mercado, para aplicação nos rejeitos de minério de ferro, visando à sua disposição por empilhamento. O estudo completo é apresentado em Guimarães (2011). filtros a vácuo (filtros de discos convencionais e filtros horizontais de correia) foi realizado através de ensaios de laboratório conhecidos como teste de folha (leaf test), com alimentação por cima. No dimensionamento, foi utilizado o método clássico estabelecido por Dahlstrom e Silverblatt (1977). Para outras tecnologias, foram utilizadas metodologias próprias dos fabricantes de equipamentos. da lama proveniente da etapa de deslamagem (93 a 96\% da massa), o overflow da ciclonagem de adensamento dos rejeitos da concentração magnética (fluxo $-1+0,150 \mathrm{~mm})$. No geral, pode ser observado, nas lamas, um grande percentual de material retido na malha de $10 \mu \mathrm{m}(40$ a $60 \%)$.

Os rejeitos de flotação apresen- taram, em geral, um máximo de $15 \%$ do material acima de $150 \mu \mathrm{m}$ e $10 \%$ abaixo de $10 \mu \mathrm{m}\left(\mathrm{d}_{50}\right.$ variando de 70 a $90 \mu \mathrm{m})$. Observa-se uma granulometria bem mais fina no rejeito da usina de Vargem Grande $\left(\mathrm{d}_{50}=20 \mu \mathrm{m}\right)$ e, mais grossa, no rejeito de Brucutu $\left(\mathrm{d}_{50}=130 \mu \mathrm{m}\right)$, embora com participação significativa de lamas.

\section{Comparativo das tecnologias de filtragem para os rejeitos de flotação}

A Figura 2 apresenta um gráfico comparativo dos resultados obtidos de dimensionamento dos filtros, para os rejeitos de flotação (excluídas Brucutu e Vargem Grande, por apresentarem taxas unitárias de filtragem e umidade das tortas muito diferentes, se comparadas com as demais usinas).

O filtro de disco convencional apresentou boa taxa unitária de filtragem, com valor próximo a $1.300 \mathrm{~kg} / \mathrm{h} /$ $\mathrm{m}^{2}$ e umidade da torta entre 10 a $13 \%$. O filtro horizontal de correia apresentou umidade da torta com cerca de um ponto percentual menor e, aproximadamente, o dobro da taxa unitária de filtragem, isto quando se compara este filtro com o filtro de disco convencional. A diferença, na umidade da torta, verificada nos testes de bancada, entre essas duas tecnologias de filtragem (disco e correia), pode 
ser explicada pelo emprego de diferentes elementos filtrantes (exigidos nos equipamentos industriais).

O filtro cerâmico apresentou valores de taxa unitária de filtragem seme-

Figura 1

Faixa granulométrica dos principais rejeitos das usinas de ferro do Quadrilátero Ferrífero.

Figura 2

Taxa unitária de filtragem em função da umidade da torta de rejeitos de flotação, para filtro de disco convencional, filtro de disco cerâmico, filtro horizontal de correia e filtro-prensa horizontal. lhante àqueles obtidos pelo filtro de disco convencional. Os valores de umidade da torta foram, neste caso, menores, em cerca de dois pontos percentuais (entre $9 \mathrm{e}$ $11 \%)$. Menores valores de taxa unitária de filtragem, próximo a $250 \mathrm{~kg} / \mathrm{h} / \mathrm{m}^{2}$, foram observados com o emprego de filtro prensa horizontal. Esse filtro apresentou, no entanto, os menores valores de umidade de torta (entre 5 a $10 \%$ ).
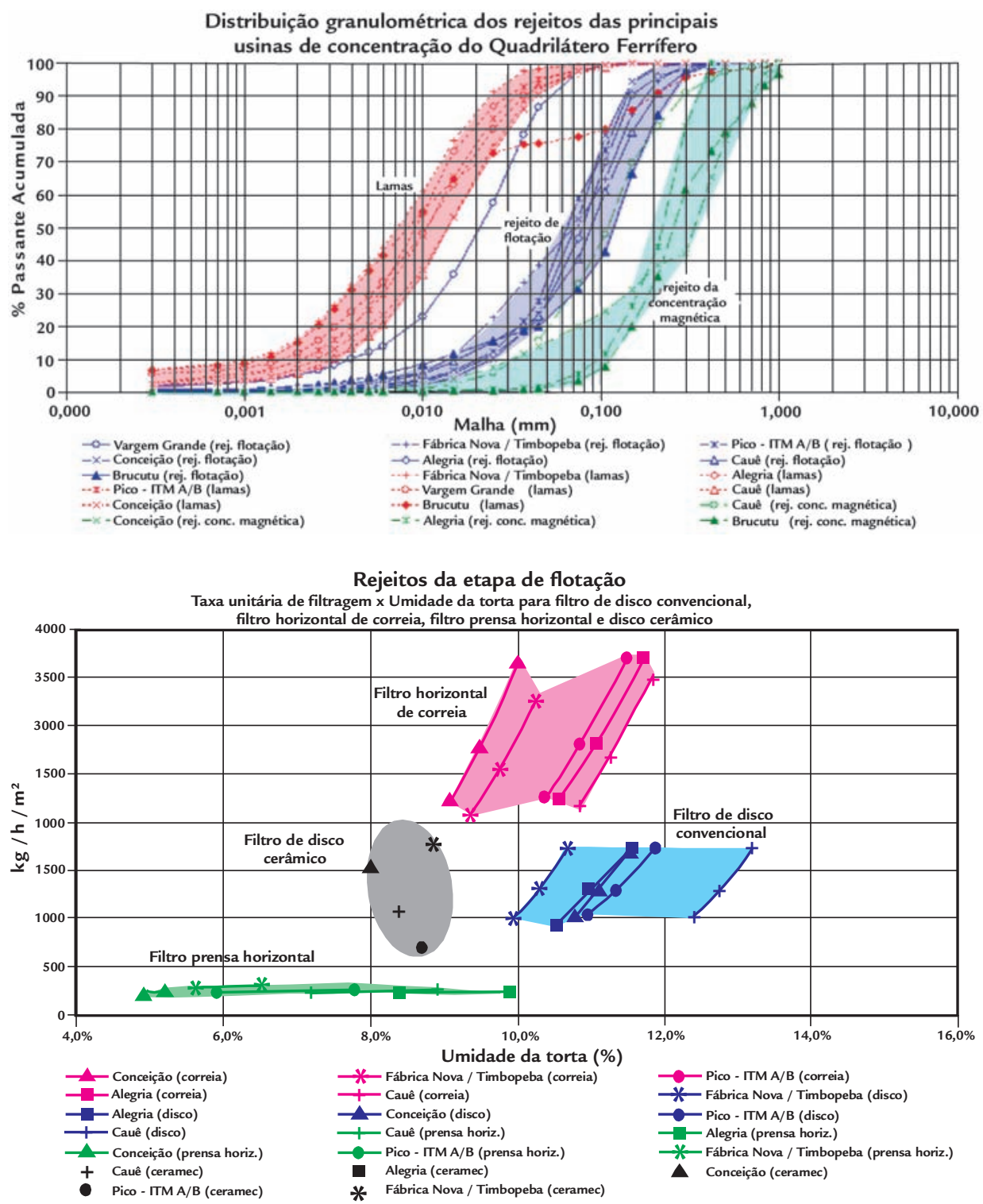

\section{Comparativo das tecnologias de filtragem para as lamas}

A filtragem de lamas só foi possível, quando nelas se aplicou a tecnologia de filtragem sob pressão (filtro-prensa horizon- tal e vertical) e hiperbárica (filtro de disco encapsulado), porém com baixa taxa unitária de filtragem $\left(<300 \mathrm{~kg} / \mathrm{h} / \mathrm{m}^{2}\right)$ e umi- dade da torta relativamente alta (próximo a $20 \%$, para os filtros sob pressão), como pode ser verificado na Figura 3.

\section{Comparativo das tecnologias de filtragem - análise econômica}

Foi feita uma estimativa dos preços dos equipamentos, incluindo bomba de vácuo e compressores, exigidos para cada tipo de filtro.

A Figura 4 mostra uma comparação entre os custos de aquisição dos equipamentos por unidade de filtragem, considerando-se: impostos $(18,05 \%)$, taxas de importação $(40 \%)$ e câmbio $(€ 1,00=\mathrm{R} \$ 2,60$; US\$ $1.00=$
R\$ 1,80). Observa-se, pela Figura 4, que o filtro de disco convencional e o de prensa horizontal apresentam o menor custo de investimento por área de filtragem. Os filtros horizontal de correia, de disco cerâmico, hiperbárico e de prensa vertical são, respectivamente, $2,9,3,6,5,3$ e 9, 8 vezes mais onerosos, por área de filtragem, que o filtro de disco convencional.
A potência necessária, para cada unidade de filtragem, na instalação, é mostrada na Figura 5. Observa-se que o consumo, por área do filtro de disco cerâmico, é semelhante ao filtro-prensa horizontal e corresponde a $15 \%$ do consumo do filtro de disco convencional, $7 \%$ do filtro horizontal de correia e cerca de $10 \%$ dos filtro de pressão vertical e hiperbárico. 
Lamas

Taxa unitária de filtragem $x$ Umidade da torta para filtros prensa horizontal, prensa vertical e hiperbárico

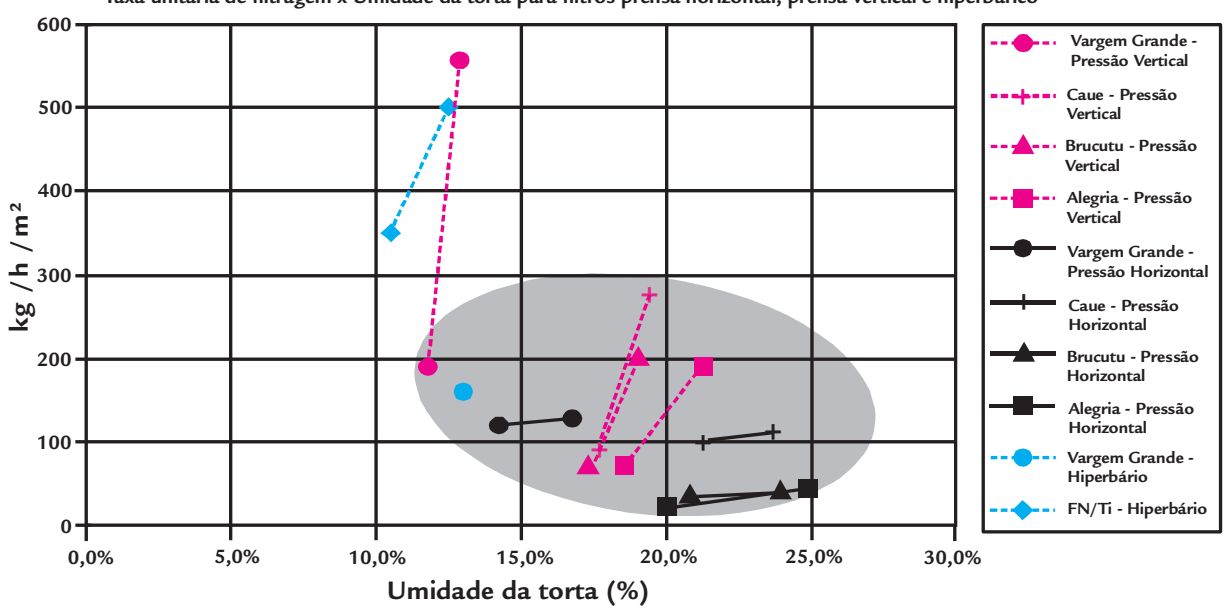

Comparativo: custos de filtragem (filtros + bombas de vácuo + compressores)

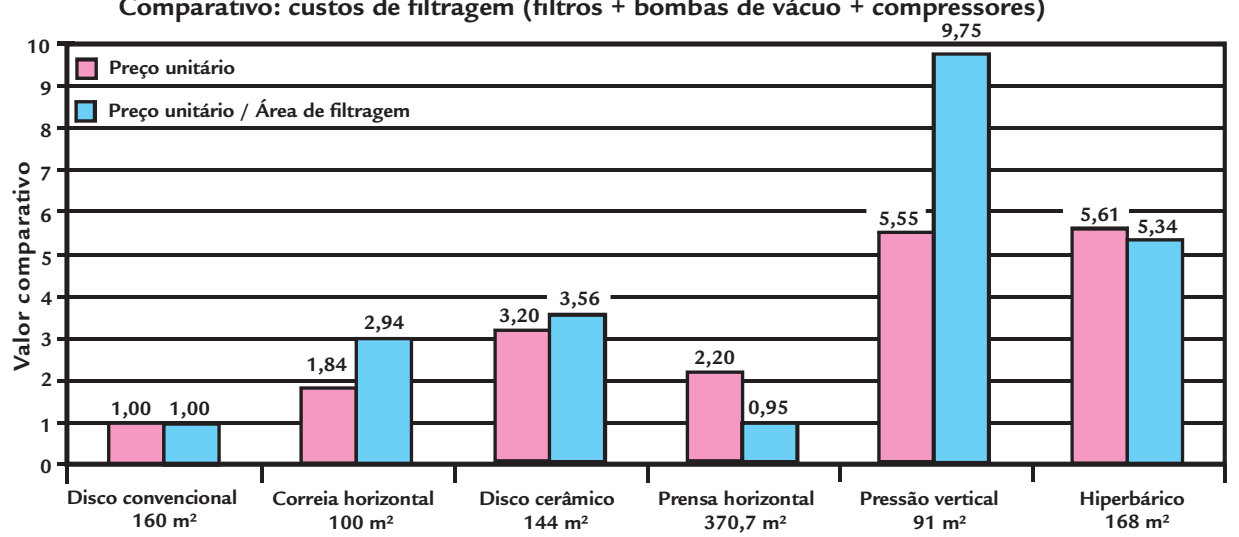

Potência por unidade de filtragem (filtros + bombas de vácuo + compressores)

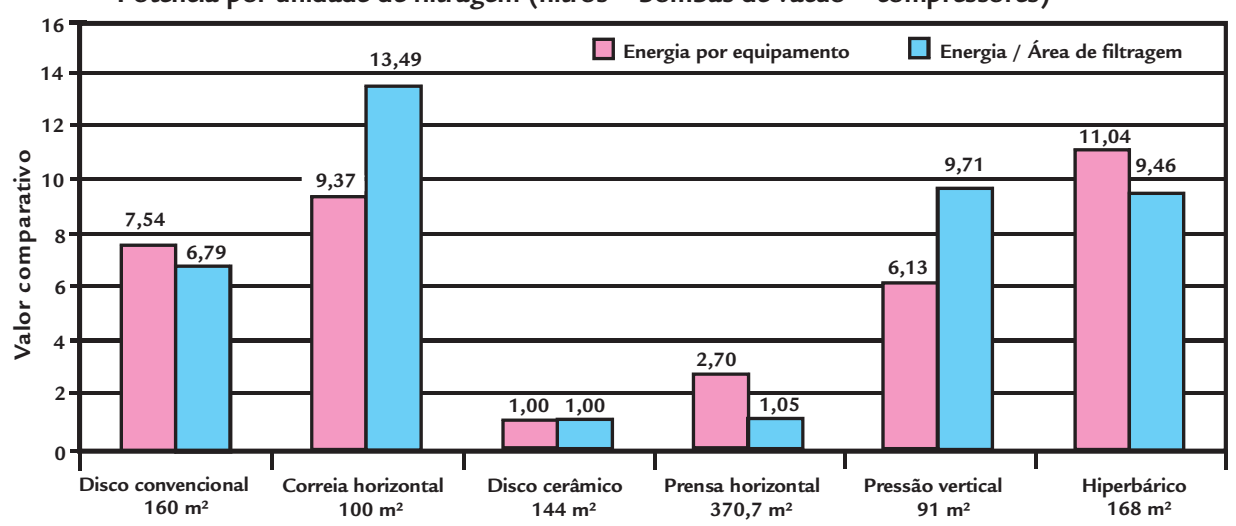

\section{Alternativa de filtragem dos rejeitos da ITM I VGR}

A Figura 6 mostra um fluxograma proposto para a filtragem de rejeitos da ITMI VGR ou projetos similares de beneficiamento de itabiritos pobres, visando-se ao empilhamento dos rejeitos. Nesse caso, procurou-se verificar a melhor alternativa técnico-econômica para a filtragem dos rejeitos e lamas.

$\mathrm{O}$ dimensionamento do filtro de disco convencional da ITMI VGR foi baseado em leaf test, tendo sido realizado em amostra gerada em planta-piloto. Os valores considerados de taxa unitária de filtragem e umidade de torta, para outros tipos de filtros, foram obtidos por extrapolação, tendo-se como base os estudos anteriores. A quantidade calculada de equipamentos, para filtragem dos rejeitos de flotação, é apresentado na Tabela 1.

Para o cálculo do Valor Presente do Desembolso (VPdesembolso), que, na prática, significa trazer para o presente, ou seja, para o tempo em que se iniciou o projeto, todas as despesas a uma determinada taxa de juros, foram consideradas as seguintes premissas: período: 20 anos; taxa de desconto: $12,0 \%$ ao ano; custo
Figura 3

Taxa unitária de filtragem, em função da umidade da torta, das lamas para filtro de prensa horizontal, prensa vertical e hiperbárico.

Figura 4

Comparativo: custo de aquisição por tipo de filtro.

Figura 5

Comparativo: potência instalada, por tipo de filtro.

de energia: 0,07537 R \$/kWh. Para esse cálculo foram considerados como CAPEX somente os custos dos equipamentos da etapa de filtragem (filtro, bombas de vácuo e compressores). Da mesma forma, para o OPEX, foram considerados apenas custos de energia elétrica. Os resultados da análise econômica, para a etapa de filtragem dos rejeitos de flotação da ITMI VGR, estão representados na Figura 7.

Pode-se concluir que a melhor alternativa técnico-econômica, para filtragem dos rejeitos de flotação, é o emprego de 
Figura 6 Fluxograma simplificado proposto para filtragem de rejeitos de flotação e lamas.
Tabela 1

Quantidade calculada de equipamentos, para filtragem dos rejeitos de flotação.

Figura 7

Comparativo: custos de filtragem dos rejeitos da ITMI VGR.

filtro de disco convencional (menor valor presente do desembolso). No entanto, o filtro de disco cerâmico apresenta baixo consumo de energia, o que pode torná-lo com menor valor presente de desembolso, dependendo do custo de energia do projeto. Para um aumento no custo de energia acima de $37 \%$ do valor adotado como premissa, o filtro de disco cerâmico apresenta menor VPdesembolso.

\section{Conclusão}

A filtragem de rejeitos feita com vista à sua disposição em pilhas mos-

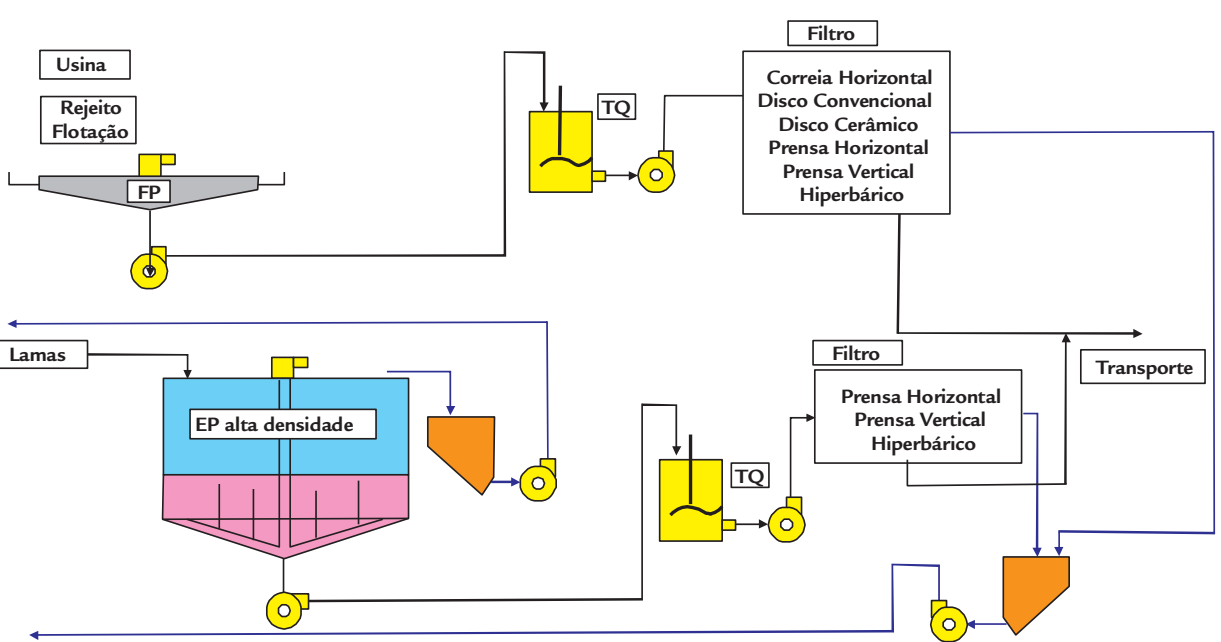

\begin{tabular}{|c|c|c|c|c|c|c|}
\hline & \begin{tabular}{|c|} 
Disco \\
Convencional \\
\end{tabular} & $\begin{array}{r}\text { Horizontal } \\
\text { de correia }\end{array}$ & \begin{tabular}{|c|} 
Prensa \\
Horizontal
\end{tabular} & Hiperbárico & \begin{tabular}{|l|} 
Pressão \\
Vertical \\
\end{tabular} & \begin{tabular}{|c|} 
Disco \\
Cerâmico
\end{tabular} \\
\hline $\begin{array}{l}\text { Taxa unitária } \\
\text { de filtragem } \\
\left(\mathrm{kg} / \mathrm{h} / \mathrm{m}^{2}\right)\end{array}$ & $1.036,6$ & $2.200,0$ & 288,0 & $2.900,0$ & $2.500,0$ & $1.000,0$ \\
\hline $\begin{array}{c}\text { Umidade } \\
\text { da torta (\%) }\end{array}$ & 12,0 & 10,7 & 8,0 & 10,0 & 10,0 & 8,6 \\
\hline $\begin{array}{l}\text { Área total de } \\
\text { filtragem }\left(\mathrm{m}^{2}\right)\end{array}$ & 1.187 & 559 & 4.270 & 424 & 492 & 1.230 \\
\hline $\begin{array}{c}\text { Área de cada } \\
\text { filtro }\left(\mathrm{m}^{2}\right)\end{array}$ & 160 & 100 & 370,7 & 168 & 91 & 144 \\
\hline $\begin{array}{l}\text { Quantidade } \\
\text { de filtros }\end{array}$ & 8 & 6 & 12 & 3 & 6 & 9 \\
\hline
\end{tabular}

Massa de sólidos projeto: $1.025 \mathrm{t} / \mathrm{h}$ (nominal) x 1,2 (fator de projeto).

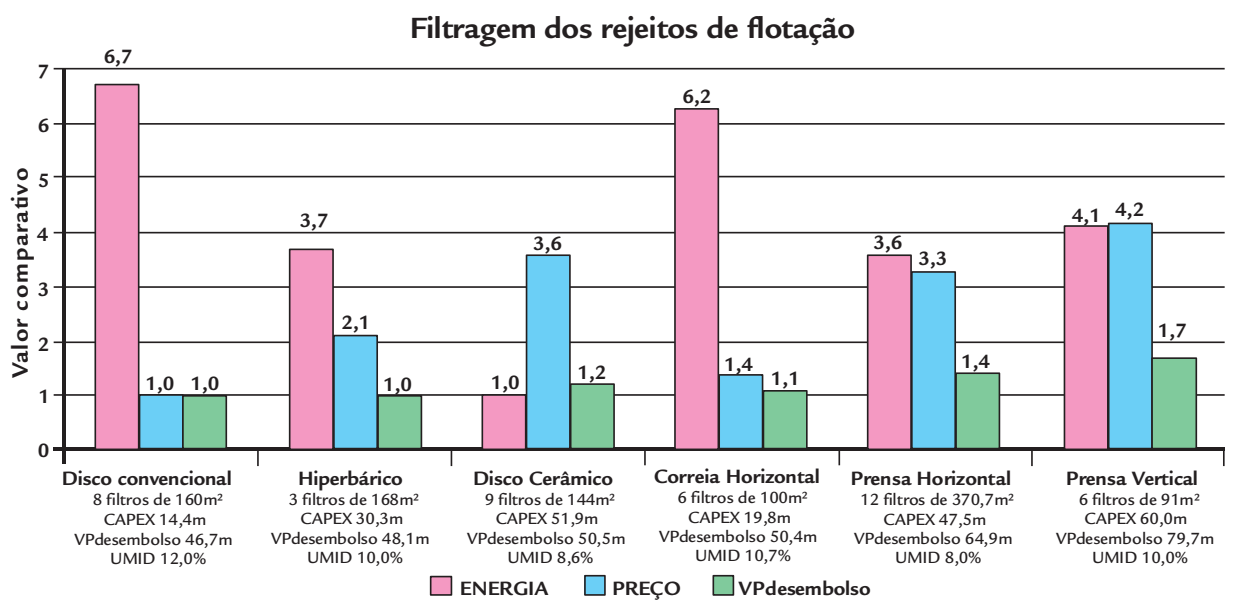

Para o projeto ITMI VGR, não foram geradas lamas para realização de ensaios de filtragem. Para esse estudo conceitual, adotou-se, como taxa unitária de filtragem e umidade da torta, a média dos resultados obtidos com as lamas das usinas testadas (Tabela 2).

A Figura 8 mostra os resultados de análise econômica, para a etapa de filtragem das lamas (mantendo as mesmas

premissas adotadas anteriormente para o cálculo do valor presente do desembolso). Verifica-se que a melhor alternativa técnico-econômica, para filtragem de lamas, é o emprego de filtro-prensa horizontal (menor valor presente de desembolso), embora tal filtro apresentasse, nos ensaios de bancada, a mais baixa taxa unitária de filtragem (média de $\left.85,8 \mathrm{~kg} / \mathrm{h} / \mathrm{m}^{2}\right)$. trou ser uma alternativa viável ao método tradicional de disposição de rejeitos diluídos em barragens. A melhor opção técnico-econômica para 
filtragem dos rejeitos de flotação é com filtro de disco convencional ou filtro de disco cerâmico (a escolha depende, principalmente, do custo de energia). A filtragem das lamas, também, mostrou ser tecnicamente possível em bancada (necessário teste-piloto), sendo o uso de filtro-

\begin{tabular}{c|c|c|c}
\hline & Prensa Horizontal & Hiperbárico & Pressão Vertical \\
\hline Taxa unitária de filtragem $\left(\mathbf{k g} / \mathbf{h} / \mathbf{m}^{2}\right)$ & 85,8 & 292,5 & 307 \\
\hline Umidade da torta $\mathbf{( \% )}$ & 20,9 & 12,3 & 18,2 \\
\hline Área total de Filtragem $\left.\mathbf{( m}^{\mathbf{2}}\right)$ & 7.633 & 2.240 & 2.134 \\
\hline Área de cada filtro $\left.\mathbf{( m}^{\mathbf{2}}\right)$ & 370,7 & 168 & 91 \\
\hline Quantidade de filtros & 21 & 14 & 24 \\
\hline
\end{tabular}

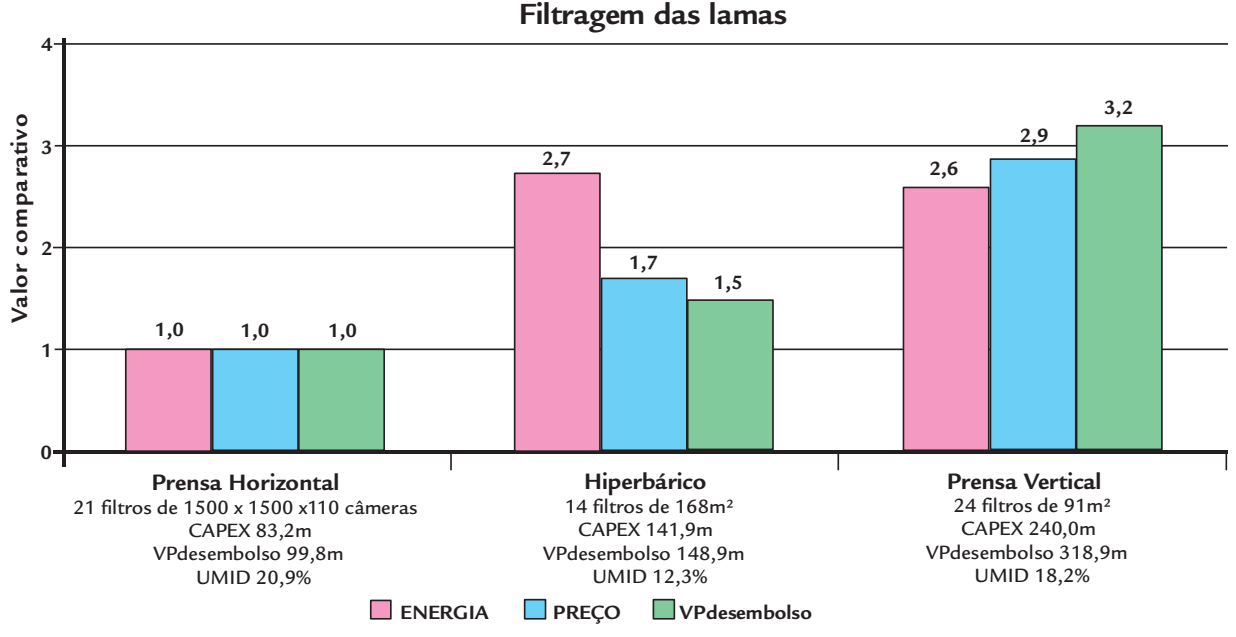

\section{Referências Bibliográficas}

BAGINSK, L.F. (Líder Sênior Licenciamento Ambiental - VALE). Mensagem recebida por <vania.andrade@vale.com> em 28 mai. 2009.

DAHLSTRON, D.A., SILVERBLATT, C.E. Continuous vacuum and pressure filtration. In: Purchas, D. B. (ed). Solid/liquid separation and scale up. Croydon Uplands Press. 1977. p. 445-491.

GUIMARÃES, N.C. Filtragem de rejeitos de minério de ferro visando a sua disposição em pilhas. Belo Horizonte: UFMG, 2011. 114 p. (Dissertação de Mestrado).

LEDUC, M., SMITH, M.E. Tailings Co Disposal - Innovation for Cost Saving and Liability Reduction. The Latin American Mining Record. July/Aug. 2003.

Tabela 2

Cálculo do número de filtros para lamas - Projeto ITMI VGR.

prensa horizontal a melhor escolha econômica, embora o filtro de pressão vertical apresente taxa unitária de filtragem 3,5 vezes superior.

Figura 8

Comparativo: custos de filtragem das lamas da ITMI VGR.

Artigo recebido em 01 de junho de 2011. Aprovado em 31 de julho de 2012. 